\title{
Determinación de los parámetros de austenización intercrítica para el desarrollo de aceros multifásicos mediante análisis dilatométrico
}

\author{
Determination of intercritical austenization \\ parameters for development of multiphase \\ steel using dilatometric analysis
}

Paula Andrea Pérez Espitia ${ }^{1,2}$, Ricardo Emilio Aristizábal Sierra ${ }^{1}$, Claudia Patricia Serna Giraldo ${ }^{1}$, Dany Michel Andrade Centeno ${ }^{2}$, Hélio Goldenstein ${ }^{2}$

\footnotetext{
${ }^{1}$ Grupo de Investigaciones Pirometalúrgicas y de Materiales - GIPIMME, Departamento de Ingeniería Metalúrgica y de Materiales, Facultad de Ingeniería, Universidad de Antioquia, Medellín, Medellín, Colombia e-mail: pandrea.perez@udea.edu.co, claudia.serna@udea.edu.co,ricardo.aristizabal@udea.edu.co ${ }^{2}$ Laboratorio de Transformaciones de Fases - LTF, Departamento de Ingeniería Metalúrgica y de Materiales, Escuela Politécnica, Universidad de Sao Paulo, Sao Paulo, Sao Paulo, Brasil.

e-mail:damianc245@usp.br, hgoldens@usp.br
}

\section{RESUMEN}

Los aceros multifásicos son una familia de materiales con propiedades mecánicas excepcionales que incluyen alta resistencia mecánica, excelente ductilidad e índice de endurecimiento y buena conformabilidad. Estas propiedades están relacionadas con la microestructura, que puede ser desde combinaciones de ferrita y martensita (aceros dúplex) hasta microestructuras complejas con presencia de ferrita, bainita, martensita y austenita retenida como en el caso de los aceros TRIP. Una de las etapas fundamentales en la fabricación de estos aceros es la austenización intercrítica que se produce entre las temperaturas $\mathrm{A}_{1}$ y $\mathrm{A}_{3}$, de la cual depende la fracción de austenita de alta temperatura y la composición química de las fases, en especial el contenido de carbono. Con el fin de estudiar esta etapa del tratamiento térmico se fabricaron aceros con variaciones en la concentración de carbono $(0.2-0.5 \%)$ y silicio $(1.4-2.0 \%)$ y se realizaron ensayos dilatométricos. Los resultados permitieron establecer las temperaturas adecuadas de tratamiento térmico intercrítico de acuerdo con la fracción de austenita de alta temperatura deseada. Además, se realizó un estudio de las variaciones en la composición química de la austenita en función de la temperatura de austenización intercrítica (TAI). Los resultados se analizaron a la luz de las posibilidades para las etapas posteriores que incluyen temple o austemperado y su posible impacto en la microestructura y las propiedades mecánicas de las aleaciones tratadas térmicamente.

Palabras claves: Aceros multifásicos, Austenización intercrítica, Análisis dilatométrico.

\section{ABSTRACT}

The Multiphase steels are materials with exceptional mechanical properties including high strength, excellent ductility and hardening coefficient and good conformability. These properties are related with the microstructure, which can be a combination of ferrite and martensite (Dual phase steels) or more complex microstructures with ferrite, bainite, martensite and retained austenite as in TRIP steels. One of the most important stages on the fabrication of multiphase steels is the intercritical austenization step which is performed between the A1 and A3 temperatures. This step determines the amount and chemistry (especially carbon) of the high temperature austenite. Aiming to study the intercritical austenization step, steels with variations in carbon $(0.2-0.5 \mathrm{wt} \%)$ and silicon $(1.4-2.0 \mathrm{wt} \%)$ were manufactured and dilatometric studies were conducted. The results allowed to stablish the intercritical austenization temperature (IAT) according with the needed amount of high temperature austenite. Also, the variation in chemistry of the high temperature austenite was studied as a function of the intercritical austenization temperature. (IAT) The results were analyzed according with the possibilities of the step after austenization, i.e., quenching or austempering and its impact on the micro- 
structure and properties of the heat treated alloys.

Keywords: Multiphase steels, Intercritical annealing, Dilatometric analysis.

\section{INTRODUCCIÓN}

Los aceros multifásicos son una familia de materiales con propiedades mecánicas excepcionales que incluyen alta resistencia mecánica, excelente ductilidad e índice de endurecimiento. La combinación de estas propiedades les permite tener además, una mayor conformabilidad y a su vez el diseño de componentes más livianos, lo que disminuye los gastos energéticos asociados a su procesamiento y el consumo de combustible en industrias como la automotriz [1]. Estas propiedades están relacionadas con su microestructura, que puede ser desde combinaciones de ferrita y martensita (aceros dúplex) hasta microestructuras complejas con presencia de ferrita, bainita, martensita y austenita retenida como en el caso de los aceros TRIP. Ambos tipos de aceros son obtenidos mediante austenización intercrítica, seguida de temple para los aceros dúplex y de austemperado para los aceros TRIP. La austenización intercrítica se da entre las temperaturas críticas $\mathrm{A}_{\mathrm{C} 1}$ y $\mathrm{A}_{\mathrm{C} 3}$, de la cual depende la fracción de austenita de alta temperatura y la composición química de las fases, en especial el contenido de carbono. La microestructura inicial y la velocidad de calentamiento, determinan a su vez, el tiempo de formación y la fracción volumétrica de la austenita de alta temperatura. Speich et al, describieron las diferentes etapas de formación de la austenita en el rango intercrítico para aceros de bajo carbono con una microestructura inicial ferrítico-perlítica, en dónde la primera etapa de formación de la austenita es determinada por la disolución de la perlita y la velocidad de crecimiento es definida principalmente por la difusión del carbono en la austenita, con una distancia de difusión aproximadamente igual al espaciamiento interlaminar de la perlita. La etapa posterior de la formación de la austenita es a partir de la ferrita, y está regida principalmente por la difusión de los elementos sustitucionales tales como el silicio y el manganeso [2]. Para conocer los valores de las temperaturas críticas, existen expresiones en función de la composición química de [3]-[5], pero éstas no incluyen variables tan determinantes en el tratamiento térmico intercrítico como la velocidad de calentamiento. En este estudio, se utilizó el análisis dilatométrico para determinar los parámetros de temperatura y tiempo de austenización intercrítica para aceros de composición química similar a la utilizada en los aceros multifásicos. A partir de la información experimental obtenida con el análisis dilatométrico, se construyeron modelos estadísticos de regresión multilineal para relacionar las temperaturas críticas de $\mathrm{A}_{\mathrm{C} 1} \mathrm{y}_{\mathrm{C} 3}$, y los parámetros de la ecuación de Avrami en condiciones de calentamiento. A partir de estos modelos, se escogió una de las aleaciones de estudio contempladas en los modelos estadísticos y se evaluó la influencia de la temperatura austenización intercrítica en la transformación de la austenita durante el enfriamiento.

\section{MATERIALES Y MÉTODOS}

Se fabricaron cuatro grupos de aleaciones en un horno de inducción con contenidos de carbono entre 0.23$0.53 \%$ y de silicio entre $1.42 \%-2.02 \%$, la tabla 1 muestra la composición química de las aleaciones, determinada mediante espectrometría de emisión óptica en un Espectrómetro Bruker Q8 Magellan. Los aceros se fundieron en un horno de inducción y se vaciaron en una coquilla de cobre precalentada a $200^{\circ} \mathrm{C}$ mediante vaciado centrífugo. Las muestras se homogenizaron a $1000^{\circ} \mathrm{C}$ por una hora con el fin de eliminar la microestructura as-cast.

Tabla 1: Composición química de las aleaciones estudiadas, \%peso.

\begin{tabular}{l|c|c|c|c}
\hline ALEACIÓN & CARBONO & SILICIO & MANGANESO & HIERRO \\
\hline \multirow{2}{*}{ BAJO C-BAJO Si } & 0.23 & 1.59 & 0.49 & \\
\cline { 2 - 4 } & 0.26 & 1.49 & 0.56 \\
\hline \multirow{2}{*}{ BAJO C-ALTO Si } & 0.25 & 1.93 & 0.51 & \multirow{2}{*}{ Bal. } \\
\cline { 2 - 4 } & 0.26 & 2.02 & 0.56 & 0.52 \\
\multirow{2}{*}{ ALTO C-BAJO SI } & 0.49 & 1.42 & 0.55 & \\
\cline { 2 - 4 } & 0.50 & 1.45 & 0.53 & \\
\hline \multirow{2}{*}{ ALTO C-ALTO SI } & 0.49 & 1.99 & 0.50 & \\
\cline { 2 - 4 } & 0.53 & 1.80 & &
\end{tabular}

Las muestras homogenizadas se sometieron a ensayos de dilatometría a dos velocidades de calenta- 
miento $0.5^{\circ} \mathrm{C} / \mathrm{s}$ y $5.0^{\circ} \mathrm{C} / \mathrm{s}$, en un dilatómetro DIL $805 \mathrm{~A} / \mathrm{D}$ en atmósfera de helio. Los cuerpos de prueba fueron de $4 \mathrm{~mm}$ de diámetro y $10 \mathrm{~mm}$ de longitud. Los ensayos de dilatometría permitieron determinar las temperaturas de inicio $\left(\mathrm{A}_{\mathrm{C} 1}\right)$ y finalización $\left(\mathrm{A}_{\mathrm{C} 3}\right)$ de la austenita, así como la fracción volumétrica de austenita formada en función del tiempo. Esta información se utilizó para construir modelos de regresión estadística multilineal para calcular las temperaturas $\mathrm{A}_{\mathrm{C} 1}$ y $\mathrm{A}_{\mathrm{C} 3}$ en función de la velocidad de calentamiento y la composición química. Para los modelos, se verificó el cumplimiento de los supuestos de normalidad, homocedasticidad e independencia, se evaluaron las interacciones de primer y segundo orden y se utilizó un alfa de 0.05 . También se determinaron los parámetros de la ecuación de Avrami para los aceros estudiados y se analizó estadísticamente la influencia de las variables de estudio en los valores de n y K (Ecuación 1). Los modelos y análisis estadísticos se realizaron en MINITAB 17.1.0.

$$
\mathrm{f}_{\gamma}=1-\exp \left[-\mathrm{kt}^{\mathrm{n}}\right]
$$

Donde $f_{\gamma}$ corresponde a la fracción volumétrica de la austenita, $\mathrm{k}$ es constante, t es el tiempo transcurrido desde el inicio hasta la finalización de la formación de la austenita, y $\mathrm{n}$ es el llamado exponente de Avrami, el cual depende de la temperatura [6].

Para estudiar el efecto de la etapa de austenización intercrítica en la transformación de la austenita durante el enfriamiento, se escogieron dos temperaturas de austenización intercrítica para una aleación Bajo carbono-Alto silicio. Las muestras se calentaron en el dilatómetro (DIL805A/D) a una velocidad de $0.5^{\circ} \mathrm{C} / \mathrm{s}$ hasta las temperaturas seleccionadas y se sostuvieron durante 5 minutos, tras lo cual se enfriaron a una velocidad de $100 \mathrm{C} \% \mathrm{~s}$ con el fin de determinar la temperatura de inicio de la martensita. Las muestras se prepararon metalográficamente mediante técnicas tradicionales y se observaron en el microscopio óptico y en el microscopio electrónico de barrido (MEB). Se determinó la fracción volumétrica de martensita mediante conteo de puntos de acuerdo a la norma ASTM E562 [7], se analizaron suficientes campos de visión hasta obtener una exactitud relativa de $10 \%$ o menor. También se determinó la composición química semicuantitativa de las fases ferrita y martensita mediante espectroscopía de energía dispersiva (EED) en el MEB. El porcentaje de carbono en la austenita de alta temperatura se estimó asumiendo que todo el carbono se particiona hacia la austenita durante la etapa de austenización intercrítica, lo cual es un supuesto válido si se tiene en cuenta el carácter adifusional de la transformación martensítica y las altas tasas de enfriamiento utilizadas durante los experimentos. Con la información obtenida se construyeron los diagramas TTT de la austenita de alta temperatura utilizando el programa MAP_STEEL_MUCG83 [8], lo cual permitió determinar las temperaturas de transformación martensítica $\mathrm{M}_{\mathrm{S}}$ y de inicio de la bainita $\left(\mathrm{B}_{\mathrm{S}}\right)$ y estimar los tiempos de transformación bainítica.

\section{RESULTADOS Y DISCUSIÓN}

Para todas las aleaciones estudiadas, la microestructura de partida de los cuerpos de prueba para los ensayos de dilatometría, corresponde a una microestructura ferrítico-perlítica, tal como se muestra en la figura 1.

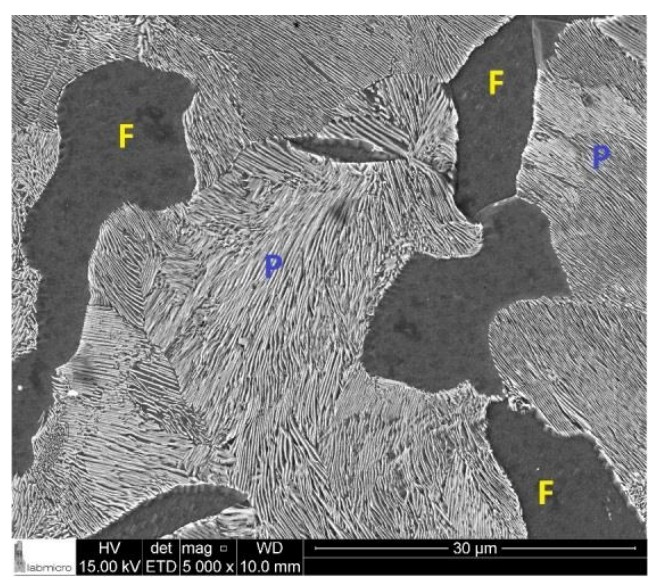

Figura 1: Micrografía de la microestructura de partida de los cuerpos de prueba para los ensayos de dilatometría, caracte- 
rística de las aleaciones de estudio; F: Ferrita, P: Perlita.

\subsection{Austenización intercrítica}

La figura 2 muestra curvas de calentamiento típicas de los materiales estudiados, en donde se incluye la primera derivada dL/dT. A partir de las curvas dilatométricas, se determinaron las temperaturas críticas de $\mathrm{A}_{\mathrm{C} 1} \mathrm{y}$ $\mathrm{A}_{\mathrm{C} 3}$. Las fracciones volumétricas de la austenita fueron determinadas a partir de la regla de fases [9].

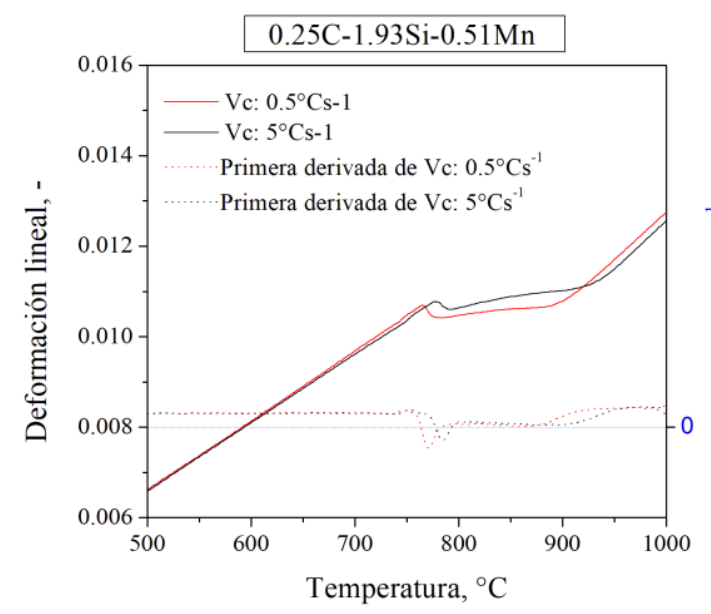

(a)

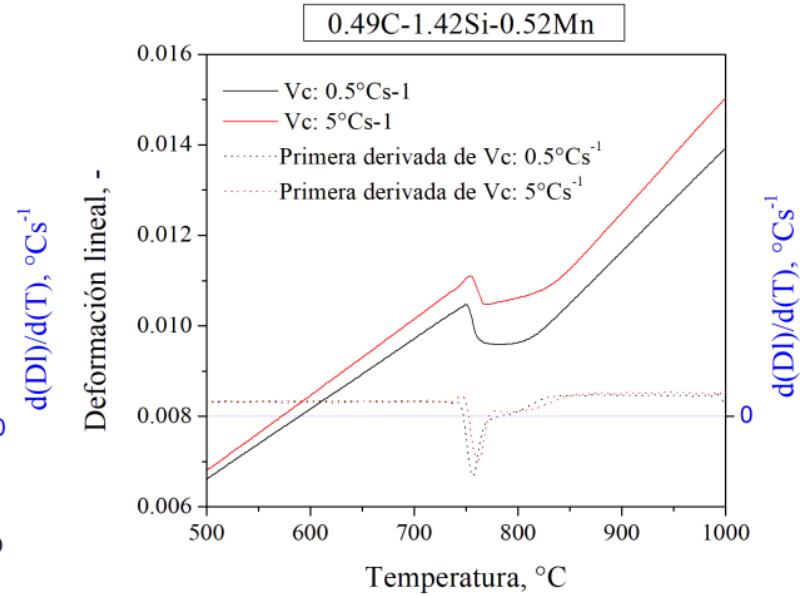

(b)

Figura 2: Curvas de dilatometría típicas de los materiales estudiados a velocidades de calentamiento, $\mathrm{Vc}: 0.5^{\circ} \mathrm{Cs}^{-1}$ y a $\mathrm{Vc}: 5^{\circ} \mathrm{Cs}^{-1}$. (a) Bajo carbono - alto Silicio, (b) Alto carbono-bajo silicio.

La temperatura $\mathrm{A}_{\mathrm{C} 1}$ es punto de la curva deformación vs temperatura, en dónde la deformación se desvía de su comportamiento lineal y empieza a descender; corresponde al inicio de la formación de la austenita (temperatura $\mathrm{A}_{\mathrm{C} 1}$ ). Esta etapa de descenso corresponde a la formación de la austenita a partir de la disolución de la perlita, la cual ocurre primero debido a que depende de la difusión de los átomos de carbono. A continuación hay un cambio en la pendiente que coincide con el mínimo de la primera derivada, la cual se hace cero cuando la perlita termina de disolverse [5] y comienza la formación de la austenita por el consumo de los granos ferríticos, lo cual implica difusión de los átomos sustitucionales como el silicio y el manganeso y por lo tanto es más lenta. Finalmente, la curva comienza a ascender nuevamente de manera lineal indicando el final de la transformación (temperatura $\mathrm{A}_{\mathrm{C} 3}$ ).

El análisis estadístico mostró que, para las aleaciones estudiadas, la temperatura $\mathrm{A}_{\mathrm{C} 1}$ depende de la concentración de silicio y de la velocidad de calentamiento, mientras que la temperatura $\mathrm{A}_{\mathrm{C} 3}$ depende del porcentaje de carbono, silicio, manganeso y de la velocidad de calentamiento. Las ecuaciones 2 y 3 corresponden a los modelos estadísticos de regresión multilineal obtenidos para $\mathrm{A}_{\mathrm{C} 1}$ y $\mathrm{A}_{\mathrm{C} 3}$ y la figura 3 muestra la comparación entre los valores calculados y los experimentales. La tabla 1 muestra los resultados obtenidos para las aleaciones evaluadas.

$$
\begin{aligned}
& \mathrm{Ac} 1=706.56+1.801(\mathrm{Vc})+32.33(\mathrm{Si}) \\
& \mathrm{Ac} 3=822.0+4.277(\mathrm{Vc})-331.7(\mathrm{C})+43.32(\mathrm{Si})+199.9(\mathrm{Mn})
\end{aligned}
$$




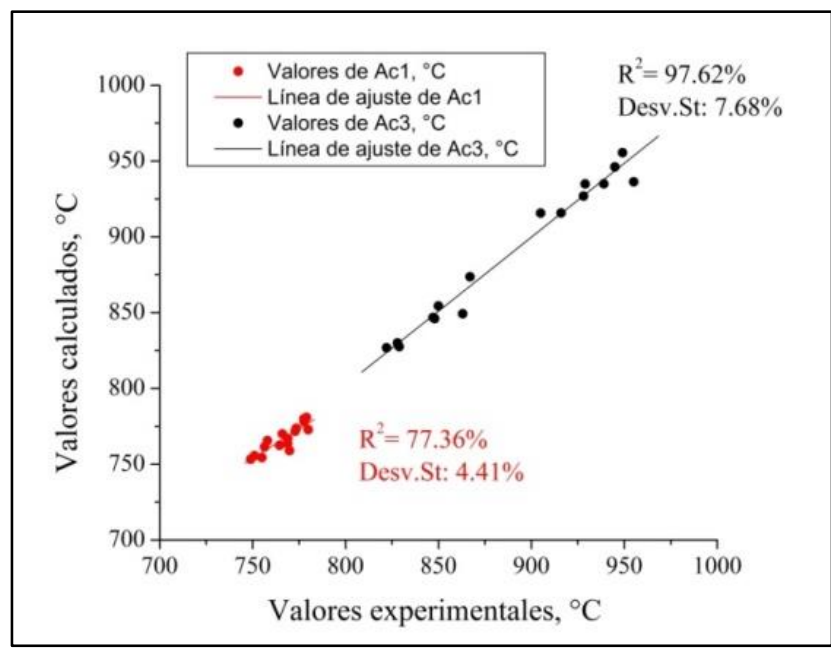

Figura 3: Gráfico de comparación de los valores calculados con los valores experimentales para las temperaturas $\mathrm{A}_{\mathrm{Cl}} \mathrm{y}$ $\mathrm{A}_{\mathrm{C} 3}$.

Tabla 2: Resultados de la determinación de las temperaturas $\mathrm{A}_{\mathrm{C} 1}$ y $\mathrm{A}_{\mathrm{C} 3}$.

\begin{tabular}{|c|c|c|c|c|c|c|c|c|}
\hline \multirow{3}{*}{ ALEACIÓN } & \multirow{3}{*}{$\begin{array}{c}\text { VELOCIDAD } \\
\text { DE CALEN- } \\
\text { TAMIENTO, } \\
{ }^{\circ} \mathrm{C} / \mathrm{S}\end{array}$} & \multirow{3}{*}{ C } & \multirow{3}{*}{$\mathbf{S i}$} & \multirow{3}{*}{ Mn } & \multicolumn{4}{|c|}{ TEMPERATURA, ${ }^{\circ} \mathrm{C}$} \\
\hline & & & & & \multicolumn{2}{|c|}{ EXPERIMENTAL } & \multicolumn{2}{|c|}{ CALCULADA } \\
\hline & & & & & $\mathrm{A}_{\mathrm{C} 1}$ & $\mathrm{~A}_{\mathrm{C} 3}$ & $\mathrm{~A}_{\mathrm{Cl}}$ & $\mathrm{A}_{\mathrm{C} 3}$ \\
\hline \multirow{4}{*}{$\begin{array}{c}\text { Bajo carbono } \\
\text { bajo silicio }\end{array}$} & 0.5 & \multirow{2}{*}{0.23} & \multirow{2}{*}{1.59} & \multirow{2}{*}{0.49} & 769.88 & 915.98 & 758.89 & 915.70 \\
\hline & 5.0 & & & & 768.93 & 938.98 & 766.99 & 934.95 \\
\hline & 0.5 & 026 & 140 & 056 & 751.02 & 905.01 & 755.56 & 915.61 \\
\hline & 5.0 & 0.20 & 1.49 & 0.50 & 768.86 & 928.92 & 763.66 & 934.85 \\
\hline \multirow{4}{*}{$\begin{array}{c}\text { Bajo carbono } \\
\text { alto silicio }\end{array}$} & 0.5 & \multirow{2}{*}{0.25} & \multirow{2}{*}{1.93} & \multirow{2}{*}{0.51} & 766.03 & 928.06 & 769.91 & 926.84 \\
\hline & 5.0 & & & & 777.78 & 944.95 & 778.02 & 946.09 \\
\hline & 0.5 & 026 & 202 & 056 & 779.93 & 955.03 & 772.73 & 936.29 \\
\hline & 5.0 & 0.20 & 2.02 & 0.50 & 779.01 & 949.06 & 780.83 & 955.53 \\
\hline \multirow{4}{*}{$\begin{array}{l}\text { Alto carbono } \\
\text { bajo silicio }\end{array}$} & 0.5 & \multirow{2}{*}{0.49} & \multirow{2}{*}{1.42} & \multirow{2}{*}{0.52} & 749.03 & 828.92 & 753.20 & 827.49 \\
\hline & 5.0 & & & & 756.48 & 847.07 & 761.30 & 846.74 \\
\hline & 0.5 & 050 & 145 & $\Omega$ & 754.98 & 827.98 & 754.26 & 829.94 \\
\hline & 5.0 & 0.50 & $1.4 \mathrm{~J}$ & $0.5 J$ & 764.54 & 863.05 & 762.37 & 849.18 \\
\hline \multirow{4}{*}{$\begin{array}{l}\text { Alto carbono } \\
\text { alto silicio }\end{array}$} & 0.5 & \multirow{2}{*}{0.49} & \multirow{2}{*}{1.99} & \multirow{2}{*}{0.53} & 772.97 & 849.94 & 771.76 & 854.35 \\
\hline & 5.0 & & & & 777.58 & 866.94 & 779.86 & 873.60 \\
\hline & 0.5 & \multirow{2}{*}{0.53} & \multirow{2}{*}{1.80} & \multirow{2}{*}{0.50} & 758.03 & 822.07 & 765.55 & 826.77 \\
\hline & 5.0 & & & & 773.51 & 847.99 & 773.65 & 846.02 \\
\hline
\end{tabular}

Para determinar los parámetros de la ecuación de Avrami para las aleaciones estudiadas se linearizaron los resultados de fracción de austenita vs tiempo de los ensayos de dilatometría, construyendo gráficos de $\ln (-\ln (1-f \gamma))$ vs $1 / t$. La pendiente de la línea recta corresponde al exponente de Avrami $(n)$ y el intercepto corresponde a $\ln (k)$. La figura 4 a muestra las gráficas linealizadas para dos de las aleaciones evaluadas, la figura 4b muestra la comparación entre los datos experimentales de fracción volumétrica de austenita en función del tiempo y los obtenidos con la ecuación de Avrami para las mismas aleaciones. La tabla 3 resume los resultados para todas las aleaciones de $n, k$ y el ajuste de la regresión $\left(\mathrm{R}^{2}\right)$. En todos los casos los ajustes de la regresión fueron mayores a 0.87 , llegando en algunos casos a 0.98 , lo cual indica una buena correlación entre los datos experimentales y la función utilizada. 


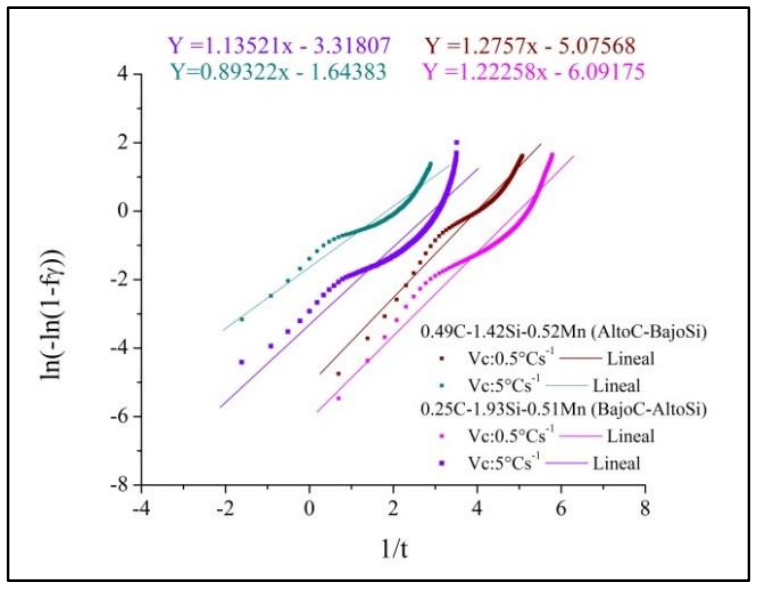

(a)

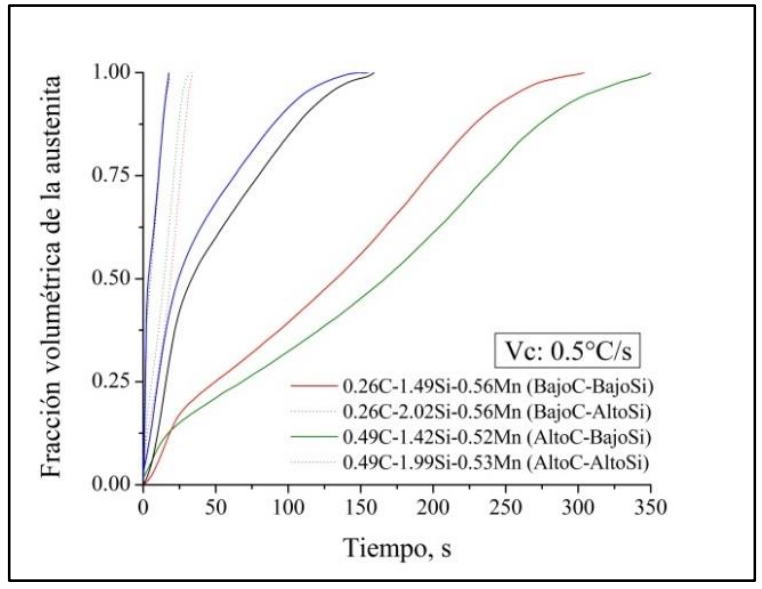

(b)

Figura 4: Determinación de los coeficientes de la ecuación de Avrami.

Tabla 3: Parámetros de la ecuación de Avrami para las aleaciones estudiadas.

\begin{tabular}{|c|c|c|c|c|c|c|c|}
\hline ALEACIÓN & $\begin{array}{l}\text { VELOCIDAD } \\
\text { DE CALEN- } \\
\text { TAMIENTO }\end{array}$ & C & Si & Mn & $\mathbf{n}$ & K & $\mathbf{R}^{2}$ \\
\hline \multirow{4}{*}{$\begin{array}{c}\text { Bajo carbono } \\
\text { bajo silicio }\end{array}$} & 0.5 & \multirow{2}{*}{0.23} & \multirow{2}{*}{1.59} & \multirow{2}{*}{0.49} & 1.12 & $4.35 \mathrm{E}-03$ & 0.91 \\
\hline & 5.0 & & & & 1.15 & $2.19 \mathrm{E}-02$ & 0.89 \\
\hline & 0.5 & \multirow{2}{*}{0.26} & \multirow{2}{*}{1.49} & \multirow{2}{*}{0.56} & 1.29 & $1.81 \mathrm{E}-03$ & 0.93 \\
\hline & 5.0 & & & & 1.23 & 3.67E-02 & 0.92 \\
\hline \multirow{4}{*}{$\begin{array}{c}\text { Bajo carbono } \\
\text { alto silicio }\end{array}$} & 0.5 & \multirow{2}{*}{0.25} & \multirow{2}{*}{1.93} & \multirow{2}{*}{0.51} & 1.22 & $2.29 \mathrm{E}-03$ & 0.92 \\
\hline & 5.0 & & & & 1.13 & $3.63 \mathrm{E}-02$ & 0.89 \\
\hline & 0.5 & \multirow{2}{*}{0.26} & \multirow{2}{*}{2.02} & \multirow{2}{*}{0.56} & 1.06 & $4.29 \mathrm{E}-03$ & 0.87 \\
\hline & 5.0 & & & & 1.14 & $3.20 \mathrm{E}-02$ & 0.88 \\
\hline \multirow{4}{*}{$\begin{array}{l}\text { Alto carbono } \\
\text { bajo silicio }\end{array}$} & 0.5 & \multirow{2}{*}{0.49} & \multirow{2}{*}{1.42} & \multirow{2}{*}{0.52} & 1.28 & $6.13 \mathrm{E}-03$ & 0.97 \\
\hline & 5.0 & & & & 0.89 & $1.93 \mathrm{E}-01$ & 0.94 \\
\hline & 0.5 & 0.50 & 145 & 055 & 1.15 & $1.27 \mathrm{E}-02$ & 0.97 \\
\hline & 5.0 & 0.50 & $1.4 \mathrm{~J}$ & 0.55 & 1.01 & $1.55 \mathrm{E}-01$ & 0.95 \\
\hline \multirow{4}{*}{$\begin{array}{l}\text { Alto carbono } \\
\text { alto silicio }\end{array}$} & 0.5 & \multirow{2}{*}{0.49} & \multirow{2}{*}{1.99} & \multirow{2}{*}{0.53} & 1.07 & $2.03 \mathrm{E}-02$ & 0.95 \\
\hline & 5.0 & & & & 1.04 & $1.50 \mathrm{E}-01$ & 0.93 \\
\hline & 0.5 & \multirow{2}{*}{0.53} & \multirow{2}{*}{1.80} & \multirow{2}{*}{0.50} & 1.00 & $2.57 \mathrm{E}-02$ & 0.98 \\
\hline & 5.0 & & & & 0.95 & $2.39 \mathrm{E}-01$ & 0.97 \\
\hline
\end{tabular}

El análisis estadístico de varianza (ANOVA) de los resultados mostró una diferencia estadística significativa en el coeficiente de Avrami $(n)$ entre las aleaciones de bajo y alto carbono, mientras que la concentración de silicio y la velocidad de calentamiento no tuvieron un efecto estadístico significativo en la variación de este parámetro. El exponente de Avrami aumentó levemente al disminuir el contenido de carbono, con un promedio para las aleaciones bajo carbono de 1.17 y de 1.05 para las aleaciones de alto carbono. El análisis de varianza también mostró, que tanto el porcentaje de carbono como la velocidad de calentamiento influyen de manera estadísticamente significativa en el parámetro K, mientras que la concentración de silicio no tiene una incidencia significativa. La tabla 4 muestra los resultados del análisis de varianza para ambos parámetros n y K. Además, se realizó una regresión estadística multilineal que relaciona el parámetro K con la velocidad de calentamiento y el porcentaje de carbono con una buena correlación, que permite estimar el parámetro K para las aleaciones en estudio (Tabla 5).

Otros autores han encontrado resultados similares Oliveira et. al., hallaron también que el exponente de Avrami parece no ser afectado por la velocidad de calentamiento, además al estudiar una aleación con $0.15 \% \mathrm{C}$ determinaron un exponente de Avrami entre 1.4 y 1.5, similar al obtenido para las aleaciones estudiadas en este trabajo, teniendo en cuenta la diferencia en la concentración de carbono. Por otro lado indican 
que $\mathrm{K}$ depende de la velocidad de calentamiento encontrando diferencias de dos órdenes de magnitud al variar la velocidad de calentamiento de $0.1^{\circ} \mathrm{C} / \mathrm{s}$ a $1.0^{\circ} \mathrm{C} / \mathrm{s}$ [10].

Tabla 4: Análisis de varianza para los parámetros de la ecuación de Avrami.

\begin{tabular}{|c|c|c|c|c|c|c|}
\hline PARÁMETRO & $\begin{array}{c}\text { FUENTE DE } \\
\text { VARIABILIDAD }\end{array}$ & $\begin{array}{c}\text { GRADOS DE } \\
\text { LIBERTAD }\end{array}$ & $\begin{array}{c}\text { SUMA DE } \\
\text { CUADRADOS }\end{array}$ & $\begin{array}{c}\text { CUADRADO } \\
\text { MEDIO }\end{array}$ & $\mathbf{F}$ & $\mathbf{P}$ \\
\hline \multirow{5}{*}{$n$} & Veloc. Cal. & 1 & 0.025803 & 0.025803 & 3.20 & 0.099 \\
\hline & $\mathrm{C}$ & 1 & 0.055042 & 0.055042 & 6.83 & 0.0123 \\
\hline & $\mathrm{Si}$ & 1 & 0.015356 & 0.015356 & 1.90 & 0.193 \\
\hline & Error & 12 & 0.096737 & 0.008061 & & \\
\hline & Total & 15 & 0.192938 & & & \\
\hline \multirow{5}{*}{$K$} & Veloc. Cal. & 1 & 0.038709 & 0.038709 & 18.92 & 0.001 \\
\hline & $\mathrm{C}$ & 1 & 0.027445 & 0.027445 & 13.42 & 0.003 \\
\hline & $\mathrm{Si}$ & 1 & 0.000374 & 0.000374 & 0.18 & 0.677 \\
\hline & Error & 12 & 0.024549 & 0.002046 & & \\
\hline & Total & 15 & 0.091077 & & & \\
\hline
\end{tabular}

Tabla 5: Regresión estadística multilineal de $\mathrm{K}$ en función del carbono con un $\mathrm{R}^{2}$ de $0.96 \%$

\begin{tabular}{c|c|c|c|c|c}
\hline TÉRMINO & COEFICIENTE & $\begin{array}{c}\text { ERROR ESTÁNDAR } \\
\text { DEL COEFICIENTE }\end{array}$ & VALOR-T & VALOR-P & VIF \\
\hline Constante & 0.021 & 0.0214 & 0.10 & 0.925 & \\
\hline Veloc. Cal. & -0.02467 & 0.00603 & -4.09 & 0.001 & 9.70 \\
\hline Carbono & -0.0088 & 0.0540 & -0.16 & 0.873 & 2.49 \\
\hline Veloc. Cal.*Carbono & 0.1240 & 0.0152 & 8.15 & 0.000 & 11.19 \\
\hline
\end{tabular}

$$
\mathrm{K}=0.021-0.02467(\mathrm{Vc})-0.088(\mathrm{C})+0.1240(\mathrm{Vc} * \mathrm{Si})
$$

\subsection{Efecto de la austenización intercrítica en la transformación de la austenita durante el enfriamiento}

Para estudiar el efecto de la austenización intercrítica en la transformación de la austenita durante el enfriamiento se utilizó una aleación bajo carbono-alto silicio de composición $0.22 \% \mathrm{C}, 2.10 \% \mathrm{Si}$ y $0.53 \% \mathrm{Mn}$ y se calentó en el dilatómetro a una velocidad de $0.5^{\circ} \mathrm{C} / \mathrm{s}$. Bajo estas condiciones se calculó utilizando la ecuación 2 una temperatura $\mathrm{A}_{\mathrm{C} 1}$ de $775.35^{\circ} \mathrm{C}$, razón por la cual se seleccionaron las temperaturas de $780^{\circ} \mathrm{C}$ y $790^{\circ} \mathrm{C}$ para realizar la austenización intercrítica con un sostenimiento de 5 minutos. $\mathrm{A}_{\mathrm{C} 1}$ se determinó experimentalmente para la muestra calentada hasta $780^{\circ} \mathrm{C}$ como $775.2^{\circ} \mathrm{C}$, mientras para la muestra calentada hasta $790^{\circ} \mathrm{C}$ presentó un valor de $774.38^{\circ} \mathrm{C}$, ambos muy cercanos al valor calculado. La figura 5 muestra las micrografías obtenidas por SEM de estas dos condiciones. Se observa que la microestructura está compuesta por ferrita y martensita. No se observó perlita sin disolver. 


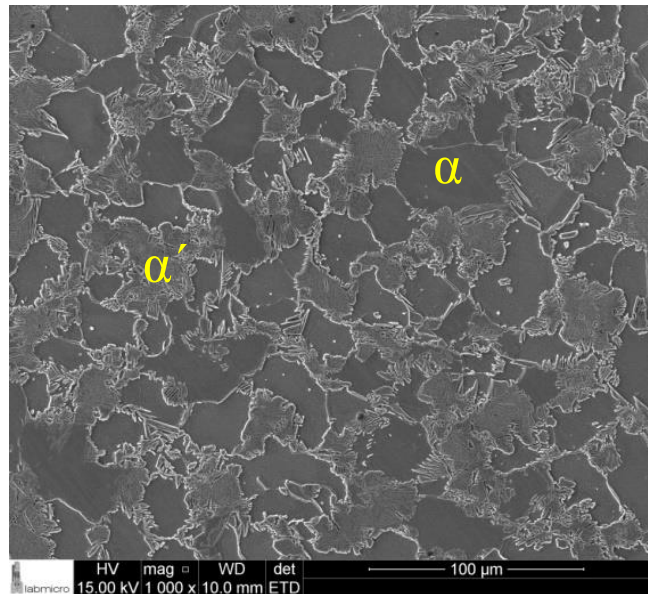

(a)

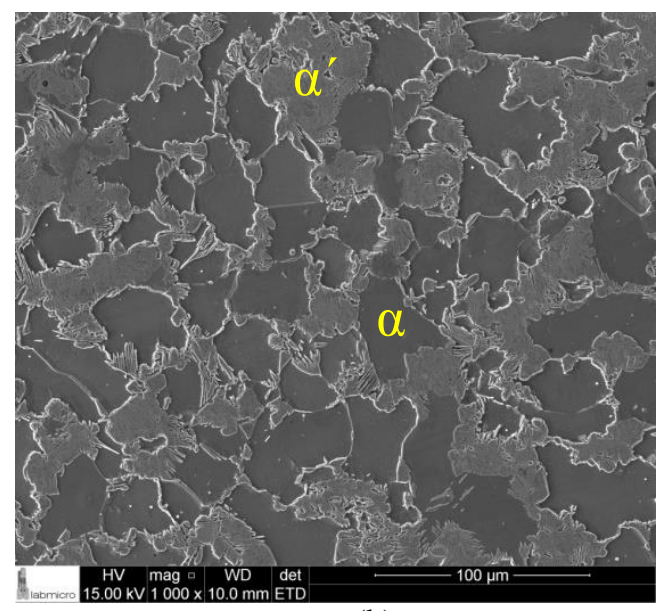

(b)

Figura 5: Micrografías del acero $0.22 \% \mathrm{C}-2.10 \% \mathrm{Si}-0.53 \% \mathrm{Mn}$ austenizado intercríticamente a: a) $780^{\circ} \mathrm{C}$ y b) $790^{\circ} \mathrm{C}$

La fracción volumétrica de la martensita fue determinada con una confiabilidad relativa inferior al $10 \%$ [11]. El contenido de carbono de la martensita fue calculado a partir de la fracción volumétrica y el contenido global de carbono, ya que la solubilidad del carbono en la ferrita es muy baja, se asumió que todo el carbono se difunde hacia la austenita. En las figuras $6 \mathrm{a}$ y $6 \mathrm{~b}$ se presentan estos resultados, en dónde se observa que los valores correspondientes a la martensita de mayor TAI, la fracción volumétrica es mayor, pero el contenido de carbono disminuye, estos valores están relacionados con los obtenidos en la microdureza dónde también es menor, asociado al contenido de carbono bajo. Para los elementos sustitucionales como el manganeso y el silicio, tanto en la ferrita como en la martensita (austenita de alta temperatura), los cuales son presentados en las figuras $6 \mathrm{c}$ y $6 \mathrm{~d}$, se observa que son similares tanto para ambas fases, cómo para ambas temperaturas de austenización intercrítica. Esto puede relacionarse con la homogeneidad que posee la microestructura inicial y a que las temperaturas a la que se austenizó intercríticamente, son muy cercanas a la temperatura de inicio de formación de la austenita $\left(\mathrm{A}_{\mathrm{C} 1}\right)$.

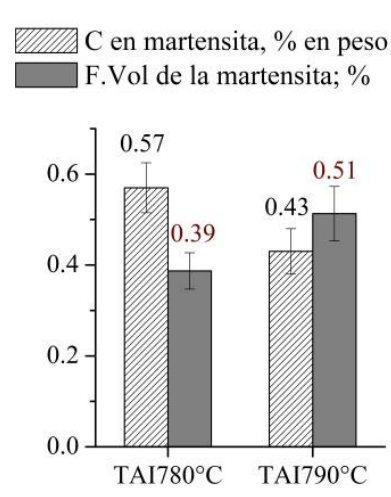

(a)

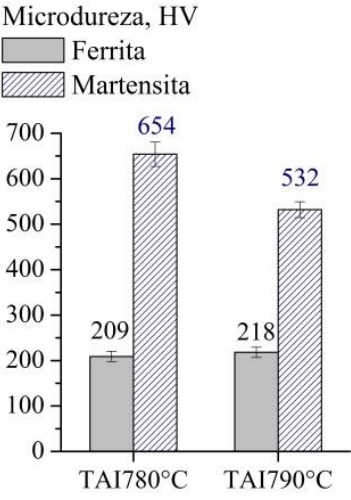

(b)

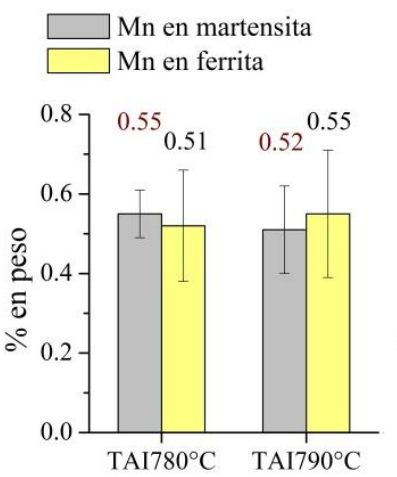

(c)

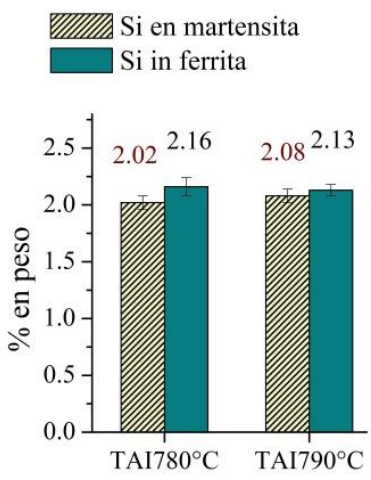

(d)

Figura 6: Para las temperaturas de austenización intercrítica evaluadas $\mathrm{TAI} 780^{\circ} \mathrm{C}$, TAI790 ${ }^{\circ} \mathrm{C}$ del acero $0.22 \% \mathrm{C}$ $2.10 \% \mathrm{Si}-0.53 \% \mathrm{Mn}$ : a) Valores de fracción volumétrica y contenido de carbono de la martensita, b) Valores de Microdureza de la ferrita y de la Martensita. Valores obtenidos por EED en la martensita y en la ferrita de c) Manganeso y d) silicio.

Con el fin de estimar las temperaturas de formación de las fases dadas en condiciones de enfriamiento, para realizar tratamientos posteriores seguidos de austenización intercrítica, se construyeron los diagramas de transformación a partir de los valores de carbono, silicio y manganeso obtenidos, a través del programa MAP Steel MUCG83 [8]. Los diagramas para las aleaciones austenizadas intercríticamente se muestran en las figuras $7 \mathrm{a}\left(780^{\circ} \mathrm{C}\right)$ y $7 \mathrm{~b}\left(690^{\circ} \mathrm{C}\right)$. Se observa que las temperaturas de Bs y Ms para la temperatura de aus- 
tenización intercrítica de $780^{\circ} \mathrm{C}$ son menores comparadas con las de $790^{\circ} \mathrm{C}$. Esto puede relacionarse con el mayor contenido de carbono de la TAI $780^{\circ}$. Si bien ambas temperaturas de austenización intercrítica difieren solamente en $10^{\circ} \mathrm{C}$, las temperaturas de $\mathrm{Bs}$ y Ms se incrementan casi en $50^{\circ} \mathrm{C}$ de diferencia.

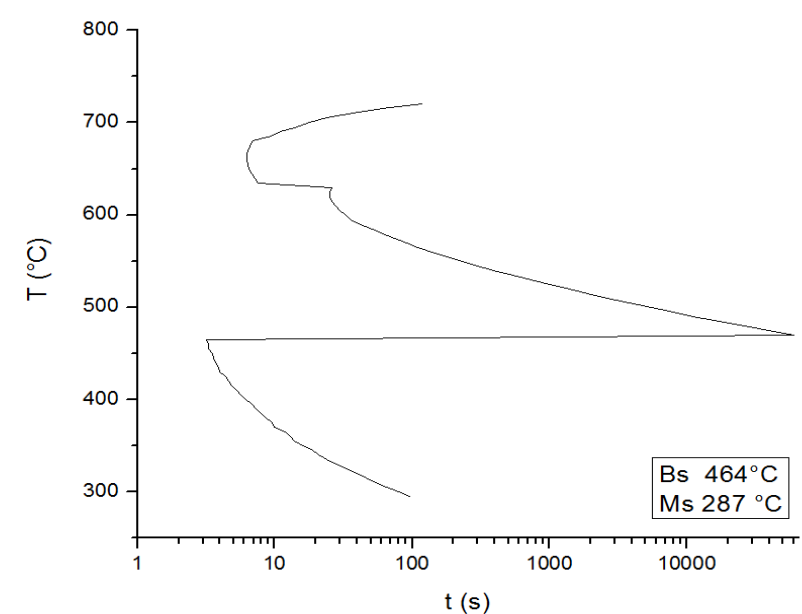

a)

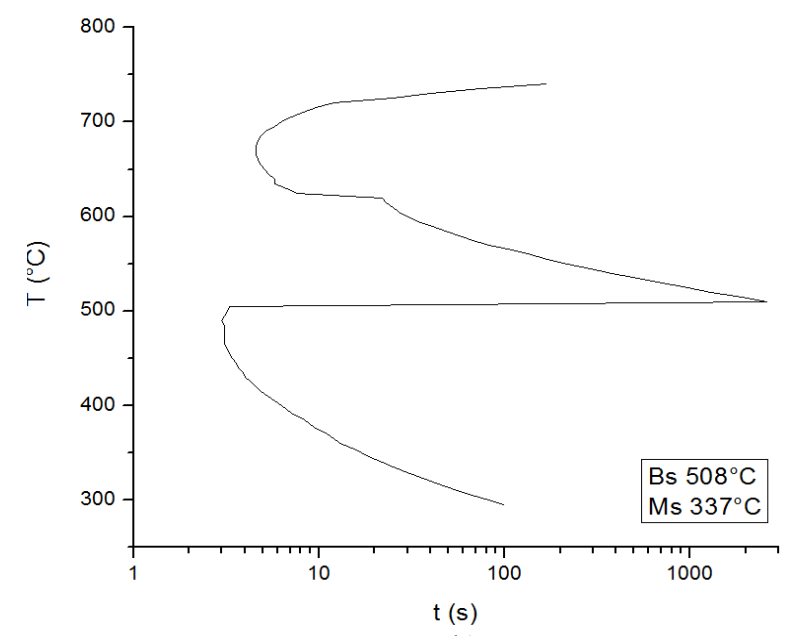

b)

Figura 7: Diagramas de transformación del acero $0.22 \% \mathrm{C}-2.10 \% \mathrm{Si}-0.53 \% \mathrm{Mn}$ construidos con la composición química de la austenita de alta temperatura formada por austenización intercrítica a temperaturas de a) $780^{\circ} \mathrm{C} \mathrm{y} \mathrm{b)} 790^{\circ} \mathrm{C}$.

\section{CONCLUSIONES}

Se construyeron modelos de regresión multilineales en dónde los factores de estudios (composición química, velocidad de calentamiento) permitieron explicar el comportamiento de las variables respuesta (temperaturas críticas) y parámetros de la ecuación de Avrami.

- Para las condiciones estudiadas se encontró que la temperatura $\mathrm{Ac}_{1}$ depende de la concentración de silicio y de la velocidad de calentamiento, mientras que la temperatura $\mathrm{Ac}_{3}$ depende del porcentaje de carbono, silicio, manganeso y de la velocidad de calentamiento.

- El análisis estadístico de varianza (ANOVA) de los resultados mostró una diferencia estadística significativa en el coeficiente de Avrami (n) entre las aleaciones de bajo y alto carbono, mientras que la concentración de silicio y la velocidad de calentamiento no tuvieron un efecto estadístico significativo en la variación de este parámetro. El ANOVA también mostró, que tanto el porcentaje de carbono como la velocidad de calentamiento influyen de manera estadísticamente significativa en el parámetro $\mathrm{K}$, mientras que la concentración de silicio no tiene una incidencia significativa

- Se escogieron los valores de las temperaturas de austenización intercrítica a partir de los modelos construidos y se evalúo la influencia de esta temperatura en una de las aleaciones de estudio, pudiendo así determinar las condiciones para tratamientos térmicos de etapas posteriores tales como temple y austemperado a bajas temperaturas.

\section{AGRADECIMIENTOS}

Los autores agradecemos a la Vicerrectoría de Investigación de la Universidad de Antioquia, por la financiación de los proyectos CODI-Convocatoria programática 2014-1038 y Estrategia de Sostenibilidad UdeaA2014-2015, los cuales enmarcan esta investigación. También agradecemos a los Laboratorios de Fundición de la Universidad de Antioquia y al de Transformaciones de fases de la Universidad de Sao Paulo, en ambos se realizó todo este estudio.

\section{BIBLIOGRAFÍA}

[1] DEMERI, M. Advanced high-strength steels. Science, Technology and applications, ASM International, 2013. pp. 264. ASM International, 2013.

[2] SPEICH, G. R., DEMAREST, V. A., MILLER, R. L. "Formation of Austenite During Intercritical Annealing of Dual-Phase Steels", Metallurgical and Materials Transactions A, v. 12, n. 8, 1981.

[3] ANDREWS, K. "Empirical Formulae for the Calculation of Some Transformation Temperatures", Jour- 
nal of the Iron and Steel Institute, v. 203, n. 7, pp. 721-727, 1965.

[4] KASATKIN, O. G., VINOKUR, B. B., PILYUSHENKO, V. L. "Calculation models for determining the critical points of steel”, Metal Science and Heat Treatment, v. 26, n. 1, pp. 27-31, 1984.

[5] PAWŁOWSKI, B. "Critical points of hypoeutectoid steel - prediction of the pearlite dissolution finish temperature Ac1f", Journal of Achievements in Materials and Manufacturing Engineering, v. 49, n. 2, pp. 331-337, 2011.

[6] ASADI ASADABAD, M., GOODARZI, M., KHEIRANDISH, S. "Kinetics of austenite formation in dual phase steels", ISIJ International, v. 48, n. 9, pp. 1251-1255, 2008.

[7] ASTM International, "Designation: E562 - 11. Standard Test Method for Determining Volume Fraction by Systematic Manual Point Count," pp. 1-7, 2011.

[8] PEET, M., BHADESHIA, H.K.D.H. "Program MAP_STEEL_MUCG83.” https://www.phasetrans.msm.cam.ac.uk/map/steel/programs/mucg83.html.

[9] FISHER, G. L., GEILS, R. H. "A method for obtaining quantitative dilatometric data from alloys undergoing a phase transformation", Metallography, v. 3, n. 2, pp. 229-233, 1970.

[10] OLIVEIRA, F. L. G., ANDRADE, M. S., COTA, A.B. "Kinetics of austenite formation during continuous heating in a low carbon steel", Materials Characterization, v. 58, n. 3, pp. 256-261, 2007.

[11] ASTM International, ASTM E562 - 11 Standard Test Method for Determining Volume Fraction by Systematic Manual Point Count. 2011. 